\title{
Estudo dos fatores motivacionais e orientação de vida de universitários
}

\author{
study of the motivational factors and life orientation \\ of college students
}

Luciane Arantes da Costa', José Roberto Andrade do Nascimento Junior ${ }^{1}$, Alexandre Igor Araripe Medeiros¹,2, Lenamar Fiorese Vieira ${ }^{1}$

Universidade de Fortaleza (UNIFOR), Fortaleza, CE, Brasil.

2Grupo de pesquisa em Educação Física, Promoção de Saúde e Esportes (UNIFOR), Fortaleza, CE, Brasil.

Recebido em: julho 2015 / Aceito em: agosto 2015

alexandreararipe@hotmail.com

\section{RESUMO}

Objetivo: investigar o nível de motivação acadêmica e o tipo de orientação de vida de universitários. Método: fizeram parte do estudo 119 acadêmicos do curso de Educação Física de uma instituição de ensino superior do estado do Paraná, sendo 77 (64,7\%) do curso integral e $42(35,3 \%)$ do curso noturno. Como instrumentos foram utilizados a Escala de Motivação Acadêmica e o Teste de Orientação de Vida. Para análise dos dados foram utilizados os testes Kolmogorov-Smirnov, alfa de Cronbach e Mann-Whitney $(\mathrm{p}<0,05)$. Resultados: os acadêmicos apresentaram moderado nível de motivação nas regulações: introjetada, identificada, integrada e intrínseca; os acadêmicos do período integral apresentaram maior regulação externa para os aspectos sociais em detrimento aos acadêmicos do período noturno $(p=0,000)$; os acadêmicos que optaram pelo bacharelado são mais regulados externamente (social) em detrimento aos estudantes de licenciatura $(p=0,000)$; os universitários, do último ano, estavam mais desmotivados $(p=0,008)$ em relação aos estudantes que recém ingressaram no ensino superior; os alunos do primeiro ano estavam mais motivados intrinsecamente $(p=0,038)$ e menos pessimistas $(p=0,048)$; os acadêmicos otimistas apresentaram maior identificação (regulação identificada) com a vida acadêmica em detrimento aos pessimistas. Considerações finais: a motivação extrínseca para os aspectos sociais prevaleceu nos acadêmicos que realizam o curso de bacharelado e no início do curso os acadêmicos são mais motivados intrinsecamente; os universitários otimistas se apresentaram mais identificados (regulação identificada) com a vida acadêmica.

Palavras-chave: Motivação; Orientação de vida; Universitários.

\section{ABSTRACT}

Objective: investigate the level of academic motivation and the life orientation of college students. Method: the subjects were 119 college students of the Physical Education course from an university of the state of Paraná, being 77 (64,7\%) of the full-time course and $42(35,3 \%)$ of the evening course, 67 (56,3\%) of the first year and $53(43,7 \%)$ of the last year. The instruments used were the Academic Motivation Scale and the Life Orientation Test. For data analysis, it was used the Kolmogorov-Smirnov test, Cronbach's alpha and Mann-Whitney test $(p<0,05)$. Results: the students had moderate levels of motivation in the introjected, identified, integrated and intrinsic regulations; the full-time students had higher external regulation for the social aspects to the detriment of the evening academics $(p=0,000)$; the academics who chose bachelor's degree are more externally regulated (social) rather than undergraduate students $(p=0,000)$; last year students were more unmotivated $(p=0,008)$ compared to students who have recently entered to the university; the first-year students were more intrinsically motivated $(p=0,038)$ and less pessimistic $(p=0,048) ;$ optimistic academics showed greater identification (identified regulation) with the academic life over the pessimists. Closing remarks: that extrinsic motivation for social conduct prevailed in the academic of bachelor's program and at the beginning of the course, students are more intrinsically motivated; the optimistic students were more identified (identified regulation) with the academic life.

Keywords: Motivation; Life Orientation; College students. 


\section{INTRODUÇÃO}

A compreensão do comportamento dos indivíduos tem sido uma preocupação da área da psicologia e desvendar o modo como os acadêmicos iniciam e se mantém no ensino superior é um desafio para os pesquisadores. De acordo com dados do Inep (Instituto Nacional de Estudos e Pesquisas Educacionais Anísio Teixeira), entre 2001 e 2005, aproximadamente 22\% dos estudantes universitários brasileiros iniciavam a graduação e desistiam ao longo do curso. ${ }^{1}$ Os cursos de graduação com maior índice de evasão compreendem a área de ciências exatas, em que, segundo Simas, ${ }^{2}$ os alunos não conseguem acompanhar as disciplinas mais difíceis que ocorrem no início do curso, como cálculo ou matemática. Entre 2001 e 2005 o curso de Educação Física se encontrou entre os quatro cursos com maiores índices de desistência no Brasil, ficando atrás apenas dos cursos de Processamento da Informação, Marketing e Ciências da Computação. ${ }^{3}$

Apesar deste dado preocupante não foram encontradas pesquisas, no cenário científico nacional, sobre os aspectos relacionados à motivação acadêmica e sua influencia no processo de desistência dos acadêmicos. No contexto internacional, estudos relacionados à motivação de universitários têm investigado aspectos relacionados ao gênero, ${ }^{4}$ aos traços de personalidade ${ }^{5,6}$ e prevenção de doenças. ${ }^{7}$ Estes aspectos aliados à orientação de vida de universitários na área da Educação Física poderiam apontar aspectos relevantes relativos à desistência dos alunos, sendo esta a lacuna que o presente estudo pretende explorar.

O estudo da motivação apresenta diferenciação entre a motivação intrínseca e extrínseca. Na motivação intrínseca, o comportamento é motivado pela satisfação ou prazer em realizar a própria atividade. Na motivação extrínseca, ao contrário, as atividades são realizadas para conseguir algum benefício externo. Embora estes aspectos tenham sido investigados, focando principalmente na motivação intrínseca, a partir dos anos de 1980, atualmente, os fatores que envolvem os diversos tipos de motivação têm sido estudados, tendo como pressuposto teórico, a Teoria da Autodeterminação (TAD). ${ }^{8}$

A TAD postula que todo ser humano é dotado de uma propensão inata para desenvolver sua própria determinação. As pessoas envolvem-se em atividades que lhes possibilitem a satisfação de necessidades psicológicas básicas: competência, autonomia e relacionamento. Para a TAD, existem três formas de comportamentos relacionados à motivação: desmotivado, motivado extrinsecamente (regulação externa, introjetada, identificada e integrada) e motivado intrinsecamente (regulação intrínseca). ${ }^{8}$

Outro constructo evidenciado nesse estudo é o otimismo, vinculado à Psicologia Positiva, ${ }^{9}$ que estuda as emoções positivas, os traços positivos do caráter e os relacionamentos positivos. ${ }^{10}$ Investigações têm demonstrado que pessoas otimistas lidam com doenças e situações desagradáveis, de forma mais apropriada, facilitando o convívio doloroso com estas situações. ${ }^{11-13}$

Assim, a motivação e o otimismo parecem ser constructos fundamentais que devem ser levados em consideração para realizar com sucesso as atividades relacionadas ao contexto universitário, influenciando o processo de ensino aprendizagem (competência), faci- litando o relacionamento entre os acadêmicos (relacionamento), e possibilitando que os acadêmicos possam tomar decisões apropriadas (autonomia). Estes fatores, evidenciados na TAD, podem determinar a continuidade e o interesse pelo curso de graduação realizado.

Em face dessas considerações, o objetivo do estudo foi investigar os fatores motivacionais e a orientação de vida de universitários de um curso de Educação Física de uma instituição de ensino superior do Estado do Paraná, buscando especificamente comparar a motivação e a orientação de vida em função do turno (integral ou noturno), da área (Licenciatura ou Bacharelado), do ano do curso (primeiro ou último ano), além de comparar o nível de motivação dos acadêmicos, em função do tipo de orientação de vida (pessimista ou otimista).

\section{MÉTODO}

Este estudo está integrado ao projeto institucional sob parecer do Comitê de Ética em Pesquisa com Seres Humanos n. 339/2011. Foram convidados a participar do estudo todos os acadêmicos do primeiro e do último ano do curso de Educação Física $(n=170)$. Aceitaram participar do estudo 119 acadêmicos de ambos os sexos, sendo que $52(43,7 \%)$ realizam o curso de licenciatura, $46(38,6 \%)$ realizam o curso de bacharelado e 21 $(17,7 \%)$ acadêmicos realizam o primeiro ano do curso, ou seja, ainda não definiram se realizarão o curso de licenciatura e bacharelado. Verificou-se que 77 (64,7\%) alunos realizam curso integral e $42(35,3 \%)$ realizam o curso noturno; 67 (56,3\%) são acadêmicos do primeiro ano (integral e noturno) e $53(43,7 \%)$ são do último ano. É importante ressaltar que na instituição investigada, o curso do período noturno é o de Licenciatura em Educação Física. A maioria dos acadêmicos participantes da investigação $(64,7 \%)$ possui de 20 a 25 anos.

Para identificar a motivação dos universitários foi utilizada a Escala de Motivação Acadêmica, ${ }^{14}$ composta de 29 itens, subdivididas em dimensões: Desmotivação, Regulação externa, Regulação externa (social), Regulação introjetada, Regulação identificada, Regulação integrada e Motivação intrínseca. As afirmativas são apresentadas em escala likert de 7 pontos (1 nada verdadeiro a 7 totalmente verdadeiro). O alfa de Cronbach geral do questionário foi $\alpha=0,54$ (Desmotivação = 0,59; Regulação externa $=0,48$; Regulação Externa (social) $=0,54$; Regulação introjetada $=0,54$; Regulação identificada $=0,40 ;$ Regulação integrada $=0,42$; Motivação intrínseca $=0,60$ ).

Para análise da orientação de vida foi utilizado o Teste de Orientação de Vida, validado para língua portuguesa por Bandeira et al. ${ }^{15}$ que avalia o constructo de otimismo em relação a eventos futuros. Possui 10 questões, sendo 3 afirmações positivas, 3 afirmações negativas e 4 neutras, que não são levadas em consideração ao se analisar o instrumento. $O$ indivíduo deve responder em função de uma escala tipo Likert de 0 (discordo totalmente) a 4 (concordo totalmente). Para as questões negativas, a pontuação é invertida $(0=4$; $1=3 ; 2=2 ; 3=1 ; 4=0$ ). O índice global de otimismo é calculado pela soma dos seis itens positivos e negativos e, quanto mais próximo de 4, maior é o grau de otimismo do indivíduo. $\mathrm{O}$ alfa de Cronbach do questionário foi $\alpha=0,87$. 
A coleta de dados foi realizada no segundo semestre de 2011 e os acadêmicos respondiam individualmente ao questionário. Participaram do estudo acadêmicos que concordaram com a investigação e assinaram o Termo de Consentimento Livre e Esclarecido.

Para análise da distribuição dos dados, utilizou-se o teste de Kolmogorov-Smirnov. Os dados não apresentaram distribuição normal, assim foram utilizados Mediana (Md) e Quartis (Q1; Q3), para caracterização dos resultados. Para a avaliação da consistência interna das dimensões dos questionários de motivação acadêmica e orientação de vida, efetuou-se o alfa de Cronbach; e para a verificação da correlação entre as dimensões dos questionários, utilizou-se o teste $U$ de Mann-Whitney, sendo adotada significância $p<0,05$.

\section{RESULTADOS}

Verificou-se (Tabela 1) que os acadêmicos do curso de Educação Física apresentaram escores moderados nas dimensões de motivação extrínsecas mais próximas da motivação intrínseca, além de moderado nível de regulação intrínseca e baixo nível de desmotivação.

Nota-se também que estes estudantes se mostraram otimistas $(M d=3,33)$ em relação à orientação de vida (Tabela 1), mas também com um índice de pessimismo considerado moderado $(M d=2,67)$. Já o índice global de otimismo dos acadêmicos foi alto $(\mathrm{Md}=3,00)$,
Tabela 1 - Nível de motivação acadêmica e orientação de vida dos acadêmicos de Educação Física.

\begin{tabular}{ll}
\hline Variáveis & Md (Q1; Q3) \\
\hline Motivação acadêmica & \\
Desmotivação & $1,33(1,00 ; 2,17)$ \\
Regulação Externa & $3,40(2,40 ; 4,60)$ \\
$\quad$ Regulação Externa (Social) & $2,75(1,75 ; 4,25)$ \\
$\quad$ Regulação Introjetada & $4,00(2,80 ; 5,00)$ \\
Regulação Identificada & $4,50(3,50 ; 5,50)$ \\
$\quad$ Regulação Integrada & $5,75(4,50 ; 6,25)$ \\
$\quad$ Regulação Intrínseca & $4,33(3,67 ; 5,67)$ \\
Orientação de vida & \\
$\quad$ Pessimismo & $2,67(2,00 ; 3,33)$ \\
$\quad$ Otimismo & $3,33(2,67 ; 3,67)$ \\
Índice Global de Otimismo & $3,00(2,50 ; 3,50)$ \\
\hline
\end{tabular}

Md: Mediana; Q1; Q3: Intervalo Interquartil.

indicando uma orientação de vida otimista (Tabela 1). A Tabela 2 apresenta a comparação do nível de motivação acadêmica e orientação de vida dos acadêmicos de Educação Física em função do turno do curso.

Houve diferença significativa $(p=0,000)$ entre os grupos na dimensão de na Regulação Externa (social), evidenciando que os acadêmicos do período integral apresentaram maior regulação externa para os aspectos sociais em detrimento aos acadêmicos do período noturno. A Tabela 3 apresenta a comparação do nível de motivação acadêmica e orientação de vida dos acadê-

Tabela 2 - Comparação do nível de motivação acadêmica e orientação de vida dos acadêmicos de Educação Física em função do turno do curso.

\begin{tabular}{|c|c|c|c|}
\hline Variáveis & $\begin{array}{c}\text { Integral }(n=77) \\
\text { Md }(01 ; 03)\end{array}$ & $\begin{array}{c}\text { Noturno }(n=42) \\
\text { Md }(01 ; 03)\end{array}$ & $p$ \\
\hline \multicolumn{4}{|l|}{ Motivação acadêmica } \\
\hline Desmotivação & $1,17(1,00 ; 2,00)$ & $1,33(1,00 ; 2,37)$ & 0,242 \\
\hline Regulação Externa & $3,40(2,30 ; 3,60)$ & $3,90(2,60 ; 5,00)$ & 0,155 \\
\hline Regulação Externa (Social) & $3,75(2,12 ; 4,60)$ & $1,75(1,18 ; 2,50)$ & $0,000 *$ \\
\hline Regulação Introjetada & $4,00(2,80 ; 4,80)$ & $4,20(3,30 ; 5,25)$ & 0,314 \\
\hline Regulação Identificada & $4,50(3,00 ; 5,50)$ & $5,00(3,88 ; 5,62)$ & 0,143 \\
\hline Regulação Integrada & $5,75(4,50 ; 6,37)$ & $5,50(4,68 ; 6,31)$ & 0,989 \\
\hline Regulação Intrínseca & $4,67(3,67 ; 5,67)$ & $4,33(2,67 ; 5,37)$ & 0,339 \\
\hline \multicolumn{4}{|l|}{ Orientação de vida } \\
\hline Pessimismo & $2,67(2,00 ; 3,33)$ & $2,67(2,33 ; 3,67)$ & 0,187 \\
\hline Otimismo & $3,33(2,83 ; 3,67)$ & $3,00(2,67 ; 4,00)$ & 0,723 \\
\hline Índice Global de Otimismo & $3,00(2,33 ; 3,50)$ & $2,83(2,50 ; 3,50)$ & 0,548 \\
\hline
\end{tabular}

Md: Mediana; Q1;03: Intervalo Interquartil. * Diferença significativa $-p<0,05$.

Tabela 3 - Comparação do nível de motivação acadêmica e orientação de vida dos acadêmicos de Educação Física em função da área do curso.

\begin{tabular}{|c|c|c|c|}
\hline Variáveis & $\begin{array}{c}\text { Licenciatura }(n=52) \\
\text { Md (Q1; Q3) }\end{array}$ & $\begin{array}{c}\text { Bacharelado }(n=46) \\
\text { Md }(01 ; 03)\end{array}$ & $p$ \\
\hline \multicolumn{4}{|l|}{ Motivação acadêmica } \\
\hline Desmotivação & $1,33(1,00 ; 2,17)$ & $1,33(1,00 ; 2,00)$ & 0,991 \\
\hline Regulação Externa & $3,80(2,60 ; 5,00)$ & $3,40(2,40 ; 4,85)$ & 0,510 \\
\hline Regulação Externa (Social) & $1,75(1,25 ; 2,50)$ & $3,50(2,00 ; 4,29)$ & $0,000 *$ \\
\hline Regulação Introjetada & $4,10(2,85 ; 5,20)$ & $4,20(2,60 ; 5,00)$ & 0,898 \\
\hline Regulação Identificada & $5,00(3,50 ; 5,50)$ & $4,50(3,00 ; 5,50)$ & 0,619 \\
\hline Regulação Integrada & $5,50(4,50 ; 6,25)$ & $5,75(4,44 ; 6,31)$ & 0,974 \\
\hline Regulação Intrínseca & $4,50(2,83 ; 5,45)$ & $4,00(3,33 ; 5,09)$ & 0,674 \\
\hline \multicolumn{4}{|l|}{ Orientação de vida } \\
\hline Pessimismo & $2,67(2,33 ; 3,67)$ & $2,67(2,00 ; 3,33)$ & 0,331 \\
\hline Otimismo & $3,33(2,67 ; 4,00)$ & $3,33(3,00 ; 3,67)$ & 0,781 \\
\hline Índice Global de Otimismo & $2,91(2,50 ; 3,50)$ & $3,00(2,50 ; 3,50)$ & 0,658 \\
\hline
\end{tabular}

Md: Mediana; Q1;03: Intervalo Interquartil. *Diferença significativa $-p<0,05$. 
Tabela 4 - Comparação do nível de motivação acadêmica e orientação de vida dos acadêmicos de Educação Física em função do ano do curso.

\begin{tabular}{|c|c|c|c|}
\hline & Primeiro Ano $(n=67)$ & Último Ano ( $n=52)$ & \\
\hline Variáveis & $\operatorname{Md}(01 ; 03)$ & Md (01; 03) & $p$ \\
\hline \multicolumn{4}{|l|}{ Motivação acadêmica } \\
\hline Desmotivação & $1,00(1,00 ; 1,83)$ & $1,50(1,05 ; 2,17)$ & $0,008^{*}$ \\
\hline Regulação Externa & $3,40(2,20 ; 4,60)$ & $3,80(2,60 ; 5,00)$ & 0,183 \\
\hline Regulação Externa (Social) & $2,50(1,50 ; 4,50)$ & $3,00(1,81 ; 4,15)$ & 0,604 \\
\hline Regulação Introjetada & $4,20(3,00 ; 5,00)$ & $4,00(2,65 ; 4,95)$ & 0,571 \\
\hline Regulação Identificada & $4,50(3,50 ; 5,50)$ & $4,50(3,12 ; 5,50)$ & 0,488 \\
\hline Regulação Integrada & $5,75(4,75 ; 6,50)$ & $5,63(4,25 ; 6,25)$ & 0,640 \\
\hline Regulação Intrínseca & $4,67(3,67 ; 6,00)$ & $4,33(3,33 ; 5,00)$ & $0,038^{*}$ \\
\hline \multicolumn{4}{|l|}{ Orientação de vida } \\
\hline Pessimismo & $2,33(2,00 ; 3,33)$ & $3,00(2,33 ; 3,58)$ & $0,048^{*}$ \\
\hline Otimismo & $3,33(2,67 ; 3,67)$ & $3,33(2,67 ; 3,67)$ & 0,741 \\
\hline Índice Global de Otimismo & $2,83(2,33 ; 3,33)$ & $3,00(2,50 ; 3,50)$ & 0,199 \\
\hline
\end{tabular}

Md: Mediana; Q1;03: Intervalo Interquartil. *Diferença significativa $-p<0,05$.

Tabela 5 - Comparação do nível de motivação dos acadêmicos de Educação Física em função do tipo de orientação de vida.

\begin{tabular}{lccc}
\hline Variáveis & $\begin{array}{c}\text { Otimista }(\mathbf{n}=\mathbf{8 3}) \\
\text { Md (01; 03) }\end{array}$ & $\begin{array}{c}\text { Pessimista }(\mathbf{n}=\mathbf{3 6}) \\
\text { Md (Q1; 03) }\end{array}$ & $\boldsymbol{p}$ \\
\hline Motivação acadêmica & & & \\
$\quad$ Desmotivação & $1,33(1,00 ; 2,00)$ & $1,08(1,00 ; 2,17)$ & 0,438 \\
$\quad$ Regulação Externa & $3,80(2,40 ; 5,00)$ & $3,10(2,25 ; 4,55)$ & 0,102 \\
$\quad$ Regulação Externa (Social) & $2,50(1,75 ; 4,25)$ & $3,30(1,75 ; 4,42)$ & 0,630 \\
Regulação Introjetada & $4,40(3,00 ; 5,00)$ & $3,80(2,65 ; 4,60)$ & 0,061 \\
Regulação Identificada & $5,00(4,00 ; 5,50)$ & $4,00(2,63 ; 5,00)$ & $0,048^{*}$ \\
Regulação Integrada & $5,75(5,00 ; 6,50)$ & $5,25(4,06 ; 6,25)$ & 0,147 \\
Regulação Intrínseca & $4,67(3,67 ; 5,67)$ & $4,33(3,08 ; 5,58)$ & 0,468 \\
\hline
\end{tabular}

Md: Mediana; Q1;03: Intervalo Interquartil. *Diferença significativa $-p<0,05$.

micos de Educação Física, em função da área do curso.

Verificou-se diferença significativa $(p=0,000)$, na dimensão Regulação Externa (social), indicando que os acadêmicos que optaram pelo bacharelado são mais regulados externamente para os aspectos sociais da vida acadêmica em detrimento aos estudantes de licenciatura (Tabela 3).

Ao comparar o nível de motivação dos acadêmicos do primeiro e do último ano (Tabela 4), encontrou-se diferença significativa na desmotivação $(p=0,008)$, evidenciando que os universitários do último ano são mais desmotivados em relação aos estudantes que recém ingressaram no ensino superior. Percebeu-se também que os alunos do primeiro ano são mais motivados intrinsecamente (regulação intrínseca), para a vida acadêmica $(p=0,038)$.

Em relação ao tipo de orientação de vida (Tabela 4), os estudantes do último ano apresentaram maior índice de pessimismo em detrimento aos alunos do primeiro ano $(p=0,048)$. A Tabela 5 apresenta a comparação do nível de motivação dos acadêmicos de Educação Física, em função do tipo de orientação de vida.

Verificou-se (Tabela 5) que os acadêmicos otimistas apresentaram maior identificação (regulação identificada) com a vida acadêmica em detrimento aos estudantes considerados pessimistas $(p=0,048)$, evidenciando que são motivados extrinsecamente para a carreira acadêmica, entretanto, se identificam e sabem da importância do ensino superior para suas vidas.

\section{DISCUSSÃO}

O objetivo do presente estudo foi investigar o nível de motivação acadêmica e o tipo de orientação de vida de universitários de Educação Física, comparando os constructos em função do turno, da área e do ano do curso. Estes aspectos, como evidenciado na literatura, podem influenciar no processo de desistência dos estudantes universitários.

Apesar dos acadêmicos que realizam o curso de Educação Física não terem evidenciado uma maior motivação intrínseca, a Regulação Integrada foi a mais evidenciada (Tabela 1); esta forma de regular o comportamento possui uma linha bastante tênue em relação à motivação intrínseca, uma vez que, na motivação extrínseca por regulação integrada, os benefícios pessoais advindos da realização da atividade, expressando atitudes que os acadêmicos realizam, pois sabem de sua necessidade e importância. ${ }^{16}$

Para os autores citados anteriormente a motivação integrada é a forma mais completa de internalização de motivação extrínseca, pois os indivíduos integram suas identificações com outros aspectos do "self", aceitando seus comportamentos, sendo estes realizados em coerência com seus valores. Assim, o que é apresentado, em princípio, como regulação externa será totalmente transformado em auto-regulação, evidenciando um comportamento autodeterminado, necessário no contexto educativo. 
A motivação integrada compartilha muitas qualidades com a motivação intrínseca, pois ambas evidenciam a autonomia dos sujeitos. Apesar da motivação integrada ser considerada uma forma de motivação extrínseca, o processo de internalização já ocorre, pois o comportamento emana da vontade e valorização dos indivíduos pela atividade. ${ }^{16} \mathrm{O}$ otimismo evidenciado (tabela 1) poderia ser reflexo desse comportamento internalizado dos sujeitos. Estudos realizados, demonstram que as pessoas otimistas resolvem seus problemas mais facilmente, evidenciando a autonomia evidente nos indivíduos mais autodeterminados. ${ }^{11-13}$

No entanto, foi observado que os acadêmicos do curso integral apresentaram maior Regulação Externa (social) (Tabela 2). A Regulação Externa é a forma mais básica e menos autônoma de motivação extrínseca, na qual a pessoa realiza suas atividades para obter ou evitar consequências externas. ${ }^{16}$ Reeve, Jang, Hardre, e Omura ${ }^{17}$ realizaram estudos em que os professores ofereceram apoio à autonomia a estudantes universitários, para o exercício da atividade de aprendizagem. Estas atividades diminuíram a motivação externa, sendo que o apoio à autonomia proporcionou regulação internalizada e mais esforço na aprendizagem na realização dos trabalhos em grupo.

Os aspectos sociais podem ter sido relevantes nos acadêmicos do curso integral, visto que a estrutura curricular deste favorece um maior relacionamento entre os acadêmicos; o contato dos alunos no curso integral é amplamente estimulado pela forma das avaliações, dos trabalhos em grupos e ainda pela distribuição de aulas que favorece diversos momentos de interação entre os acadêmicos, ao contrário do período noturno em que não existem as denominadas "aulas vagas", e os alunos possuem poucos momentos de interação fora do período de aulas. Alcará e Guimarães ${ }^{18}$ verificaram que as orientações motivacionais dos alunos do curso de biblioteconomia demonstraram uma tendência para a motivação extrínseca por regulação introjetada, identificada e para motivação intrínseca, indicando que os alunos parecem perceber a regulação do seu comportamento de forma mais autônoma do que controlada.

Verificou-se ainda que os acadêmicos que optaram pelo bacharelado são mais regulados externamente para os aspectos sociais da vida acadêmica em detrimento aos estudantes de licenciatura (Tabela 3). Em geral, os acadêmicos que optam pelo curso de bacharelado são atletas ou ex-atletas e possuem um convívio nas atividades esportivas. O relacionamento é considerado pela TAD, um dos aspectos das necessidades psicológicas básicas, favorecendo a saúde psicológica e bem-estar dos indivíduos. ${ }^{19}$ Além disso, as atividades realizadas pelos indivíduos podem se reforçadas ou desencorajadas pelo meio social, ou seja, pelos professores, colegas de turma, estrutura curricular e espaços físicos para as atividades, proporcionando uma melhor qualidade de vida dos estudantes universitários. ${ }^{20}$ Black e Deci ${ }^{21}$ realizaram estudo com estudantes universitários que receberam apoio à autonomia de seus professores. Os resultados demonstraram que os estudantes que tiveram professores que apoiavam a autonomia tiveram maior regulação autônoma para a aprendizagem ao longo do semestre, melhorando os aspectos sociais e a aprendizagem.
A percepção dos acadêmicos no início da graduação foi mais orientada ao otimismo e à motivação intrínseca (Tabela 4) em detrimento aos acadêmicos do último ano. Além disso, os alunos do último ano se apresentam mais desmotivados e pessimistas comparados aos alunos que estavam no início do curso, o que poderia revelar uma preocupação com o futuro de suas carreiras e a entrada no mercado de trabalho. Em uma investigação realizada por Costa et al., ${ }^{22} \mathrm{com}$ acadêmicos de Educação Física do último ano de uma instituição pública, evidenciou que o curso de bacharelado parece ainda não ter identidade própria, visto que as disciplinas ainda enfatizam muitos conteúdos ministrados na escola, sendo as aulas voltadas para o curso de licenciatura, o que poderia desmotivar os acadêmicos, durante a formação inicial.

Ao comparar o nível de motivação dos acadêmicos de Educação Física em função do tipo de orientação de vida, observou-se que os acadêmicos otimistas são aqueles que possuem maior regulação identificada (Tabela 5). A motivação extrínseca por Regulação Identificada ocorre em situações de reconhecimento e valorização subjacentes ao comportamento. Entretanto, é necessário evidenciar que na regulação identificada as atividades, muitas vezes, são realizadas sem que o indivíduo as considere agradáveis. Para a TAD, a orientação de causalidade relacionada à autonomia ocorre quando as pessoas agem por interesse e valorização do que está ocorrendo. Dessa forma, os acadêmicos otimistas são aqueles que parecem valorizar o curso de graduação que estão realizando, demonstrando esforço e iniciativa nas tarefas desenvolvidas.

\section{CONSIDERAÇÕES FINAIS}

Concluiu-se que o turno do curso (integral ou noturno) e a área (licenciatura ou bacharelado) podem ser elementos intervenientes na regulação externa para os aspectos sociais dos acadêmicos. Este achado revela que acadêmicos que estudam no turno integral e optam pelo curso de bacharelado são mais motivados extrinsecamente para os aspectos sociais

Além disso, percebeu-se que a motivação intrínseca para os alunos do primeiro ano do curso é maior em detrimento ao último ano do curso. Foi possível notar também que o tipo de orientação de vida pode ser um elemento interveniente no nível de motivação acadêmica, visto que universitários otimistas se apresentaram mais identificados (regulação identificada) com a vida acadêmica.

Destaca-se a necessidade de futuras intervenções, principalmente em outros cursos da área da saúde, além de estudos longitudinais que possam analisar a motivação e a orientação de vida, durante o percurso da formação de universitários.

\section{REFERÊNCIAS}

1. Filho $R$, Motejunas $P$, Hipólito $O$, Lobo $M$. A evasão no ensino superior brasileiro. Cadernos de Pesquisa 2007; 37(132): 641-9. DOI: http://dx.doi.org/10.1590/S0100-15742007000300007.

2. Simas A. As graduações campeãs de desistência 2012 [cited 05/08/2012]. Available from: Disponível em: http:// www.gazetadopovo.com.br/vidauniversidade/nocampus/ 
conteudo.phtml?id=1248860\&tit $=$ As-graduacoes campeas-de-desistencia.

3. Brasil. Ministério da Educação. Instituto Nacional de Estudos e Pesquisas Educacionais. Sinopses do ensino superior. Censos do ensino superior. Comunicações pessoais. 2011 [17/11/2011]. Available from: Disponível em: http://www. inep.gov.br.

4. Delisle M, Guay F, Senecal C, Larose S. Predicting stereotype endorsement and academic motivation in women in science programs: A longitudinal model. Learning and Individual Differences 2009; 19(4): 468-75. DOI: http://dx.doi.org/10.1016/j. lindif.2009.04.002.

5. Komarraju M, Karaub S, Schmeck R. Role of the big five personality traits in predicting college students' academic motivation and achievement. Learning and Individual Differences 2009; 19(1): 47-52. DOI: http://dx.doi.org/10.1016/j.lindif.2008.07.001.

6. Tanaka M, Mizuno K, Fukuda S, Tajima S, Watanabe Y. Personality traits associated with intrinsic academic motivation in medical students. Medical Education 2009; 43(4): 384-7. DOI: http://dx.doi.org/10.1111/j.1365-2923.2008.03279.x.

7. Furia C, Lee E, Strother L, Huang T. College students' motivation to achieve and maintain a healthy weight. Am J Health Behav 2009; 33(3): 256-63. DOI: http://dx.doi. org/10.5993/AJHB.33.3.4

8. Richard R, Deci E. Intrinsic and extrinsic motivations: classic definitions and new directions. Contemporary Educational Psychology 2000; 25(1): 54-67. DOI: http://dx.doi.org/10.1006/ ceps.1999.1020

9. Seligman E, Csikszentmihalyi M. Positive psychology: an introduction. Am Psychol 2000; 55(1): 5-14. DOI: http:// dx.doi.org/10.1037/0003-066X.55.1.5.

10. Snyder CR, Lopes SJ. Psicologia Positiva: uma abordagem científica e prática das qualidades humanas. Porto Alegre2009.

11. Kubzansky D, Sparrow D, Vokonas P, Kawachi I. Is the glass half empty or half full? A prospective study of optimism and coronary heart disease in the Normative Aging Study. Psychosom Med 2001; 63(6): 910-6. DOI: http://dx.doi. org/10.1186/s13104-015-1323-5.
12. Tindle A, Chang F, Kuller H, Manson E, Robinson G, Rosal $\mathrm{C}$, et al. Optimism, cynical hostility, and incident coronary heart disease and mortality in the Women's Health Initiative. Circulation 2009; 120(8): 656-62. DOI: http://dx.doi. org/10.1161/CIRCULATIONAHA.109.918557.

13. Kim E, Park N, Peterson C. Dispositional optimism protects older adults from stroke: The health and retirement study. Stroke 2011; 42(10). DOI: http://dx.doi.org/10.1161/STROKEAHA.111.613448.

14. Guimarães S, Bzuneck S. Propriedades psicométricas de um instrumento para avaliação da motivação de universitários. Ciências e Cognição 2008; 13: 101-13.

15. Bandeira M, Bekou V, Lott K, Rocha M. Validação transcultural do teste de Orientação de Vida (TOV-R). Estudos de Psicologia 2002; 7(2): 251-8.

16. Deci E, Ryan R. The "what" and "why" of goal pursuits: Human needs and the self-determination of behavior. Psychological Inquiry 2000; 11(4): 227-68. DOI: http:// dx.doi.org/10.1207/S15327965PLI1104_ 01.

17. Reeve $\mathrm{J}$, Jang $\mathrm{H}$, Hardre $\mathrm{P}$, Omura $\mathrm{M}$. Providing a rationale in an autonomy-supportive way as a strategy to motivate others during an uninteresting activity. Motivation and Emotion 2002; 26: 183-207.

18. Rosecler A, Guimarães S. Orientações motivacionais de alunos do curso de biblioteconomia. Psicologia Escolar Educacional 2010; 14(2): 211-20. DOI: http://dx.doi.org/10.1590/S141385572010000200004.

19. Deci E, Ryan M. Handbook of self-determination research. Rochester, NY2002.

20. Deci L, Ryan M. Intrinsic motivation and self-determination in human behavior. New York1985.

21. Black $E$, Deci $L$. The effects of student self-regulation and instructor autonomy support on learning in a collegelevel natural science course: A self determination theory perspective. Science Education 2000; 84: 740-56.

22. Costa A, Souza $M$, Barbosa-Rinaldi $P$, editors. O estágio supervisionado na formação inicial do bacharel em Educação Física. XVI Congresso Brasileiro de Ciências do Esporte e III Congresso Internacional de Ciências do Esporte; 2009; Salvador. 